\title{
A adesão livre e voluntária à luz da jurisprudência dos Tribunais Superiores no Brasil
}

Voluntary and Open Membership and the decisions of the Courts in Brazil

\author{
Mario De Conto 1 \\ Faculdade de Tecnologia do Cooperativismo - ESCOOP (Brasil)
}

Sumário: I. Introdução. II. Os Princípios Cooperativistas no Direito Internacional. III. Liberdade de Associação Cooperativa na legislação brasileira. IV. Discurso decisório do Superior Tribunal de Justiça. V. Conclusões. VI. Bibliografia.

Summary: I. Introduction. II. The Cooperative Principles and the International Law. III. Freedom of Cooperative Association on the Brazilian law. IV. Decisions of the Superior Court of Justice of Brazil. V. Conclusions. VI. Bibliography.

Resumo: O presente estudo analisa o conteúdo jurídico do Princípio da Adesão Livre e Voluntária no direito brasileiro, examinando os requisitos estabelecidos pela legislação para adesão à Cooperativa, bem como as possibilidades de restrição ao ingresso de novos associados e o discurso decisório dos tribunais superiores a respeito do tema. No Direito brasileiro, a legislação confere às Cooperativas a prerrogativa de não aceitar novos associados, mesmo diante de candidatos a associados que preencham os requisitos legais e estatutários para associarem-se, sempre que houver «impossibilidade técnica de prestação de serviços». A análise do discurso decisório do Superior Tribunal de Justiça revelou o reconhecimento, pela Corte, do Princípio Cooperativista da Adesão Livre e Voluntária, rechaçando o estabelecimento de requisitos discriminatórios e arbitrários como condições de adesão a Cooperativas.

Palavras chave: Cooperativa, Adesão, Restrições.

Abstract: This study analyzes the legal content of the Principle of Open and Voluntary membership in Brazilian law, examining the requirements established by the legislation to be a member of the Cooperative, as well as the

1 Doutor em Direito. Coordenador de Ensino, Pesquisa e Extensão da Faculdade de Tecnologia do Cooperativismo - ESCOOP. Professor de Direito Cooperativo em cursos de graduação e pós-graduação. Advogado. Gerente Jurídico do Sistema OCERGS-SESCOOP/RS. Correo electrónico: mario-deconto@ocergs.coop.br. 
possibilities of restricting the entry of new members and the decision-making of higher courts regarding the theme. In Brazilian law, the legislation gives Cooperatives the prerogative of not accepting new members, even in the case of candidates for associates who fulfill the legal and statutory requirements to associate, whenever there is «technical impossibility of providing services.» The analysis of the decision-making discourse of the Superior Court of Justice revealed the recognition by the Court of the Cooperative Principle of Open and Voluntary Adhesion, rejecting the establishment of discriminatory and arbitrary requirements as conditions of admission to Cooperatives.

Keywords: cooperative, membership, restrictions. 


\section{Introdução}

As Sociedades Cooperativas são definidas pela Aliança Cooperativa Internacional ${ }^{2}$ como associações autônomas de pessoas unidas voluntariamente para satisfazer suas necessidades e aspirações econômicas, sociais e culturais através de uma empresa de propriedade comum e gerida democraticamente ${ }^{3}$.

No Brasil, as Cooperativas são consideradas sociedades de pessoas, constituídas para prestar serviços aos seus associados. Segundo a Lei 5.764/71, que rege as Sociedades Cooperativas no país, os sócios são pessoas que se obrigam a contribuir com bens ou serviços para o exercício de uma atividade econômica, de proveito comum, sem objetivo de lucro. A legislação brasileira ao disciplinar a adesão a uma Sociedade Cooperativa a vincula à observância dos requisitos estatutários de ingresso, estes diretamente relacionados a atividade econômica - 0 objeto social- que a Cooperativa pretende desenvolver. Além disso, apresenta-se incompatível no processo de adesão à Cooperativa qualquer espécie de discriminação ou arbitrariedade.

Uma particularidade em relação à adesão a Cooperativas está na faculdade, conferida pela lei, de que a Cooperativa possa restringir a admissão de sócios sempre que houver «impossibilidade técnica da prestação de serviços». Trata-se, evidentemente, de uma restrição à livre adesão em Cooperativas que se justifica na medida em que a admissão irrestrita de sócios pode prejudicar a a capacidade da Cooperativa de prestação de serviços e, evidentemente, sua própria sustentabilidade.

2 A Aliança Cooperativa Internacional assume a missão de guardiã dos Valores e Princípios Cooperativistas, congregando 284 federações e organizações de Cooperativas em 95 países. "The International Co-operative Alliance (the Alliance) is the custodian of the Co-operative Values and Principles. The Alliance is a non-profit international association established in 1895 to advance the co-operative social enterprise model. The Alliance is the apex organisation for co-operatives worldwide, representing 284 co-operative federations and organisations across 95 countries». Aliança Cooperativa Internacional. "Guidance Notes to the Co-operative Principles». Acesso em 24 de Maio de 2017. http://ica.coop/en/blueprint-themes/identity/guidancenotes.

3 A Declaração sobre a Identidade Cooperativa apresenta a definição de Cooperativa da Aliança Cooperativa Internacional: «Definition of a Co-operative. A co-operative is an autonomous association of persons united voluntarily to meet their common economic, social, and cultural needs and aspirations through a jointly-owned and democratically-controlled enterprise». Aliança Cooperativa Internacional. «Statement on Cooperative Identity». Acesso em 24 de maio de 2017. http://ica.coop/en/whats-co-op/ co-operative-identity-values-principles 
A jurisprudência do Superior Tribunal de Justiça, ao mesmo tempo em que reconhece o conteúdo jurídico do «Princípio das Portas Abertas», apresenta entendimentos divergentes quando da compatibilização entre o preenchimento de requisitos estatutários de ingresso e a demonstração da ausência de capacidade técnica para prestação de serviços da Cooperativa.

Nesse sentido, o objeto do presente trabalho é analisar o conteúdo jurídico do Princípio da Adesão Livre e Voluntária no direito brasileiro, examinando os requisitos estabelecidos pela legislação para adesão à Cooperativa, bem como as possibilidades de restrição ao ingresso de novos associados e o discurso decisório dos tribunais superiores a respeito do tema.

\section{Os Princípios Cooperativistas no Direito Internacional}

A Declaração sobre a Identidade Cooperativa, adotada pela Aliança Cooperativa Internacional a partir do Congresso de Manchester, realizado em 1995, reflete os 150 anos de evolução do movimento cooperativista, iniciando-se com os princípios lançados pelos primeiros cooperativistas, os reconhecidos Pioneiros de Rochdale, incorporando as contribuições dos fundadores do movimento cooperativista de diversos países. A Declaração estabelece a definição de sociedade cooperativa, seus valores ${ }^{4}$ e princípios ${ }^{5}$ e possui pretensões de universalidade uma

4 A Aliança Cooperativa Internacional reconhece que as Cooperativas são fundadas nos valores da ajuda mútua, auto responsabilidade, democracia, igualdade, equidade e solidariedade, "CO-OPERATIVE VALUES: Co-operatives are based on the values of selfhelp, self-responsibility, democracy, equality, equity and solidarity. In the tradition of their founders, co-operative members believe in the ethical values of honesty, openness, social responsibility and caring for others». Aliança Cooperativa Internacional. "Statement on Cooperative Identity». Acesso em 24 de Maio de 2017. http://ica.coop/en/ whats-co-op/co-operative-identity-values-principles

5 A Aliança Cooperativa Internacional reconhece os seguintes princípios cooperativistas: $1 .^{\circ}$-Adesão voluntária e livre; $2 .^{\circ}$ - Gestão democrática; $3 .^{\circ}$-Participação econômica dos membros; $4^{\circ}$-Autonomia e independência; $5^{\circ}$-Educação, formação e informação; $6 .^{\circ}$ - Intercooperação; $7 .^{\circ}$ - Interesse pela comunidade. "COOPERATIVE PRINCIPLES: The co-operative principles are guidelines by which co-operatives put their values into practice. 1. Voluntary and Open Membership. Co-operatives are voluntary organisations, open to all persons able to use their services and willing to accept the responsibilities of membership, without gender, social, racial, political or religious discrimination. 2. Democratic Member Control. Co-operatives are democratic organisations controlled by their members, who actively participate in setting their policies and making decisions. Men and women serving as elected representatives are accountable to the membership. In primary co-operatives members have equal voting 
vez que, entre seus objetivos, está o de orientar legisladores quando da elaboração de normativos e políticas públicas atinentes a Cooperativas.

No âmbito do Direito Internacional, a Declaração de Identidade Cooperativa fora expressamente recepcionada pela Recomendação 193/2002 da Organização Internacional do Trabalho, que trata da Promoção de Cooperativas ${ }^{6}$. Com efeito, a partir do advento da referida Recomendação, pode-se afirmar que os Princípios Cooperativistas adquiriram caráter normativo na ordem jurídica internacional, notadamente em face dos Estados que compõem a Organização Internacional do Trabalho.

Apresenta-se relevante referir em relação ao Princípio Cooperativo da «Adesão Livre e Voluntária» —objeto da presente análise- que sua noção está presente desde a formulação teórica de Cooperativa elaborada pelos Pioneiros de Rochdale. Nesse particular já se apresenta a

rights (one member, one vote) and co-operatives at other levels are also organised in a democratic manner. 3. Member Economic Participation. Members contribute equitably to, and democratically control, the capital of their co-operative. At least part of that capital is usually the common property of the co-operative. Members usually receive limited compensation, if any, on capital subscribed as a condition of membership. Members allocate surpluses for any or all of the following purposes: developing their co-operative, possibly by setting up reserves, part of which at least would be indivisible; benefiting members in proportion to their transactions with the co-operative; and supporting other activities approved by the membership. 4. Autonomy and Independence Co-operatives are autonomous, self-help organisations controlled by their members. If they enter into agreements with other organisations, including governments, or raise capital from external sources, they do so on terms that ensure democratic control by their members and maintain their co-operative autonomy. 5. Education, Training and Information. Co-operatives provide education and training for their members, elected representatives, managers, and employees so they can contribute effectively to the development of their co-operatives. They inform the general public - particularly young people and opinion leaders - about the nature and benefits of co-operation. 6. Co-operation among Co-operatives. Co-operatives serve their members most effectively and strengthen the co-operative movement by working together through local, national, regional and international structures.7. Concern for Community. Co-operatives work for the sustainable development of their communities through policies approved by their members». Aliança Cooperativa Internacional. "Statement on Cooperative Identity». Acesso em 24 de Maio de 2017. http://ica. coop/en/whats-co-op/co-operative-identity-values-principles

6 «3. The promotion and strengthening of the identity of cooperatives should be encouraged on the basis of: (...) (b) cooperative principles as developed by the international cooperative movement and as referred to in the Annex hereto. These principles are: voluntary and open membership; democratic member control; member economic participation; autonomy and independence; education, training and information; cooperation among cooperatives; and concern for community». Organização Internacional do Trabalho. «Recomendação 193/2002». Acesso em 24 de Maio de 2017. http://www. ilo.org/dyn/normlex/en/f?p=NORMLEXPUB :12100:0::NO::P12100_ILO_CODE:R193. 
contribuição inovadora dos primeiros cooperativistas, uma vez que tal princípio está diretamente relacionado à Liberdade de Associação, direito que somente viria a ser internacionalmente reconhecido pela Declaração Universal dos Direitos Humanos de 19487. Sarlet refere ainda que ressalvada a experiência inglesa e norte-americana, o movimento de constitucionalização da Liberdade de Associação é fenômeno bastante recente, que remonta o período pós Segunda Guerra Mundial ${ }^{8}$.

No que atine ao seu conteúdo, relevante referir que o Princípio Cooperativo da «Adesão Livre e Voluntária» caracteriza as cooperativas como organizações voluntárias, abertas a todas as pessoas aptas a utilizar seus serviços e desejem aceitar as responsabilidades decorrentes da associação, sem discriminação de gênero, social, racial, política ou religiosa.

A análise do enunciado evidencia, inicialmente, a preocupação com a (1) voluntariedade da associação; (2) a abertura da cooperativa àqueles que sejam aptos a assumir direitos e obrigações perante a sociedade; (3) a ausência de discriminação e arbitrariedades fundadas em questões sociais, raciais, políticas, religiosas ou de gênero.

A voluntariedade de associação remete à ideia de que a decisão pelo ingresso —e, em regra, a decisão de desligar-se - de uma Cooperativa, deve ser voluntária. Não se apresenta adequada à identidade cooperativa a ideia de que a associação a uma Cooperativa seja determinada, por exemplo, como requisito para o acesso a determinada política pública. Da mesma forma, a permanência no quadro social da Cooperativa pressupõe a voluntariedade que, portanto, deve estar presente durante todo o período em que mantido vínculo associativo com a Cooperativa.

A análise da abertura da Cooperativa àqueles aptos a assumir direitos e obrigações perante a sociedade requer, nesse particular, a análise de uma característica distintiva entre as Sociedades Cooperativas

7 «Article 201 . Everyone has the right to freedom of peaceful assembly and association. 2. No one may be compelled to belong to an association. Universal Declaration of Human Rights. ORGANIZAÇÃO DAS NAÇÕES UNIDAS (ONU). «Declaração Universal dos Direitos Humanos» Acesso em 24 de Maio de 2017. http:// www.ohchr.org/EN/Professionallnterest/Pages/InternationalLaw.aspx.

8 «No plano do direito constitucional positivo, ressalvada a experiência inglesa e norte-americana, o reconhecimento da liberdade de associação, na condição de direito fundamental, é mais recente, sendo, em geral (ressalvadas algumas exceções), um fenômeno do século XX, notadamente a partir da Segunda Guerra. Atualmente, como se sabe, a liberdade de associação é amplamente reconhecida, tanto na esfera internacional quanto na esfera constitucional». SARLET, Ingo Wolfgang. Liberdade de associação cooperativa, direitos e garantias fundamentais e relações privadas na perspectiva do Supremo Tribunal Brasileiro. In: MEIRA, Deolinda Aparício Jurisprudência cooperativa comentada. Lisboa: INCM, 2012. p. 44. 
e as Sociedades Empresárias. Ao contrário de um sócio/acionista de uma Sociedade Empresária, que se trata de um investidor que ingressa com seu capital na obtenção dos dividendos decorrentes da exploração da atividade empresarial, um sócio de Cooperativa deve congregar duas características essenciais: além de aportar capital, representado por quotas partes, o sócio de cooperativa é usuário da sociedade, realizando operações com a Cooperativa no cumprimento do seu objetivo social. Trata-se do que a doutrina cooperativista reconhece como a dupla qualidade do sócio, como "proprietário e usuário». Em uma Cooperativa que tenha por objeto a industrialização de produtos lácteos, por exemplo, não basta para ser sócio a intenção de investir no negócio: é imperioso que a pessoa que pretende associar-se à Cooperativa seja um produtor de leite, uma vez que, enquanto usuário, essa é a condição necessária para que o mesmo possa realizar operações com a Cooperativa. Obviamente, a análise dos requisitos estatutários de ingresso em uma Cooperativa deve ser realizada a luz de suas características próprias, notadamente de seu objeto social.

Por fim, a ausência de discriminação fundada em questões sociais, raciais, políticas, religiosas ou de gênero reflete o compromisso das Cooperativas com a utilização de instrumentos transparentes e a vedação de arbitrariedades no processo de adesão de associados.

\section{Liberdade de Associação Cooperativa na legislação brasileira}

A Liberdade de Associação, no Direito Constitucional brasileiro é reconhecida como Direito Fundamental. Segundo o texto constitucional, «é plena a liberdade de associação para fins lícitos, vedada a de caráter paramilitar» (art. 5. ${ }^{\circ}, \mathrm{XVII}$ ) além da garantia expressa de que «ninguém poderá ser compelido a associar-se ou a permanecer associado» (art. 5. ${ }^{\circ}, \mathrm{XX}$ ).

No que concerne ao tratamento constitucional de Sociedades Cooperativas, a Constituição Federal de 1988 iniciou um novo período, denominado de Período de Autogestão, em que «a criação de associações e, na forma da lei, a de cooperativas independem de autorização, sendo vedada a interferência estatal em seu funcionamento» (art. 5. , XVIII). Além disso, a Constituição Federal reconheceu a necessidade do «adequado tratamento tributário ao ato cooperativo praticado pelas sociedades cooperativas (art. 146, III, «C») e o papel do Estado, como agente normativo e regulador da atividade econômica, ao estabelecer que «a lei apoiará e estimulará o cooperativismo e outras formas de associativismo» (art. 174, § 2..$^{\circ}$ ). 
A Lei 5.764 de 16 de dezembro de 1971, que «define a Política Nacional de Cooperativismo, institui o regime jurídico das sociedades cooperativas, e dá outras providências» estabelece como características da Cooperativa em seu art. $4 .^{\circ}$, incisos I e IX, respectivamente a "adesão voluntária, com número ilimitado de associados, salvo impossibilidade técnica de prestação de serviços» e a «neutralidade política e indiscriminação religiosa, racial e social». Além disso, o art. 29 da referida lei, dispõe que "O ingresso nas cooperativas é livre a todos que desejarem utilizar os serviços prestados pela sociedade, desde que adiram aos propósitos sociais e preencham as condições estabelecidas no estatuto, ressalvado o disposto no artigo $4 .{ }^{\circ}$, item I, desta Lei».

A partir dos dispositivos legais, pode-se dividir as obrigações legais decorrentes, a partir dos destinatários da norma:

a) Candidato a associado: (1) voluntariedade; (2) desejo de utilizar os serviços prestados pela Cooperativa; (3) adesão aos propósitos sociais; (4) preenchimento das condições estabelecidas no Estatuto;

c) Cooperativa: (1) vedação ao estabelecimento de número limite de sócios; (2) vedação ao estabelecimento de critérios e procedimentos discriminatórios ou arbitrários; (3) obrigação da associação de candidato que preencha os requisitos legais e estatutários, com a (4) possibilidade de negativa de associação em caso de impossibilidade técnica de prestação de serviços.

No que concerne às disposições legais alusivas ao Candidato a Associado, verifica-se que as mesmas não guardam maior complexidade, tratando-se basicamente, que a intenção voluntária de ingressar na Cooperativa, contraindo direitos e obrigações, esteja acompanhada do cumprimento dos requisitos estatutários de ingresso.

No que concerne às obrigações legais alusivas a Cooperativas, a legislação brasileira, além dos critérios elencados no Princípio da Adesão Livre e Voluntária, atribui à Cooperativa a possibilidade de negativa de associação em caso de impossibilidade técnica de prestação de serviços e a vedação a utilização de critérios e procedimentos discriminatórios, temas cuja complexidade tem gerado discussões nos Tribunais Superiores.

\section{O Discurso decisório do Superior Tribunal de Justiça}

A análise do discurso decisório do Superior Tribunal de Justiça, ao pronunciar-se acerca das obrigações legais impostas às Cooperativas relativas à observância da Adesão Livre e Voluntária enfatiza o Princí- 
pio das Portas Abertas como limitador ao estabelecimento de restrições discriminatórias e arbitrárias ao livre ingresso de associados.

«AGRAVO REGIMENTAL NO AGRAVO EM RECURSO ESPECIAL.

COOPERATIVA DE TRABALHO MÉDICO. INGRESSO NO QUADRO. RECUSA PELA EXIGÊNCIA DE CURSO. IMPOSSIBILIDADE. PRINCÍPIO DA LIVRE ADESÃO. ART. 4. ${ }^{\circ}$ I, DA LEI N. 5.764/71. IMPOSSIBILIDADE TÉCNICA NÃO CARACTERIZADA. ACÓRDÃO EM CONSONÂNCIA COM O ENTENDIMENTO DESTA CORTE. PRECEDENTES. SÚMULA 83/ STJ. AGRAVO IMPROVIDO. 1. O Tribunal de Justiça, ao analisar a situação fática dos autos, concluiu pela ilegalidade da exigência do aludido certificado de conclusão de curso ministrado pela Unimed Campinas. 2. Nesse contexto, a jurisprudência desta Corte destaca o princípio da 'porta-aberta', consectário do princípio da livre adesão, segundo o qual não podem existir restrições arbitrárias e discriminatórias à livre entrada de novos membros nas cooperativas. 3. Dessa forma, a conclusão alcançada pelo acórdão recorrido está alinhada à jurisprudência deste Tribunal Superior, incidindo, no ponto, a Súmula 83/STJ. 4. Agravo regimental improvido» (AREsp n. ${ }^{\circ}$ 767.502/SP, Rel. Ministro MARCO AURÉLIO BELLIZZE, Terceira Turma, DJe 4/2/2016).

«AGRAVO REGIMENTAL NOS EMBARGOS DE DECLARAÇÃO NO AGRAVO EM RECURSO ESPECIAL. CIVIL. COOPERATIVA DE TRABALHO MÉDICO (UNIMED). INGRESSO DE NOVO ASSOCIADO. RECUSA. EXIGÊNCIA DE APROVAÇÃO EM PROCESSO SELETIVO E REALIZAÇÃO DE CURSO. INCIDÊNCIA DO PRINCÍPIO DA PORTA ABERTA (LIVRE ADESÃO). 1. O ingresso nas cooperativas é livre a todos que desejarem utilizar os serviços prestados pela sociedade sendo, em regra, ilimitado o número de associados, salvo impossibilidade técnica de prestação de serviços (arts. 4. ${ }^{\circ}$, I, e 29 da Lei n. ${ }^{\circ}$ 5.764/1971). Incidência do princípio da livre adesão voluntária. 2. Pelo princípio da porta-aberta, consectário do princípio da livre adesão, não podem existir restrições arbitrárias e discriminatórias à livre entrada de novo membro na cooperativa, devendo a regra limitativa da impossibilidade técnica de prestação de serviços ser interpretada segundo a natureza da sociedade cooperativa. 3. Agravo regimental não provido. (AgRg nos EDcl no AGRESP N. ${ }^{\circ} 667.072$ - SP, Rel. MINISTRO RICARDO VILLAS BÔAS CUEVA, DJe 3/3/2016).

O conteúdo jurídico do Princípio das Portas Abertas, nesses termos, apresenta-se relacionado à ausência de restrições discriminatórias e arbitrárias. Nesse ínterim, em julgado recente do Superior Tribunal de Justiça, o estabelecimento da necessidade de aprovação em Processo Seletivo Público para admissão de associados teve sua legalidade afirmada. No entendimento do Julgador, embora o ingresso nas Cooperativas 
seja livre, devem ser preenchidas as condições previamente estabelecidas. Nas suas palavras, «no caso, para ser (associado), o apelante necessitava ser aprovado no processo seletivo, o que não ocorreu, razão pela qual o desprovimento do recurso é medida que se impõe». Nesse sentido, o estabelecimento de Processo Seletivo, desde que previsto estatutariamente, não se configura como requisito discriminatório, conforme ementa da decisão:

«RECURSO ESPECIAL. Civil. Cooperativa de trabalho Médico. Recusa. Descumprimento de exigência de condições estatutárias. Possibilidade. Expressa Previsão legal. Art. 29 da lei N. ${ }^{\circ}$ 5.764/71. Recurso especial desprovido». (RECURSO ESPECIAL N. 1.616 .034 - SP, MINISTRO PAULO DE TARSO SANSEVERINO, DJe 1/2/2017).

Apresenta-se relevante se apresenta analisar o posicionamento do STJ a respeito da «Impossibilidade Técnica de Prestação de Serviços», prevista no art. $4 .^{\circ}$, I, da Lei 5764/71.

Verifica-se que, em alguns julgados, a Impossibilidade Técnica é analisada a luz da Cooperativa. Dito de outro modo, o critério estabelecido é analisar se a Cooperativa possui capacidade técnica de, ao aceitar a adesão de novos associados, prestar-lhes serviços. Nesse diapasão, o entendimento exarado do REsp n. ${ }^{\circ} 151.858 / \mathrm{MG}$ é o de que tal impossibilidade técnica da Cooperativa deve ser demonstrada, não bastando a simples inconveniência para os atuais sócios:

«Cooperativa. Nas associações com essa natureza, a limitação ao ingresso de novos associados condiciona-se à 'impossibilidade técnica de prestações de serviços' (Lei 5.764/71, artigo 4. ${ }^{\circ}$, I). Não basta para justificá-la a simples inconveniência que possa resultar para os que já integram o quadro de cooperados» (REsp n. ${ }^{\circ}$ 151.858/MG, Rel. Ministro EDUARDO RIBEIRO, Terceira Turma, DJ de 8/9/1998).

Em julgados mais recentes, todavia, o Superior Tribunal de Justiça tem manifestado o entendimento - equivocado, ao nosso ver- de que a impossibilidade de prestação de serviços deve ser analisada em face do candidato a sócio:

«CIVIL E PROCESSO CIVIL. COOPERATIVA. UNIMED. VEDAÇÃO DE INGRESSO A NOVOS MÉDICOS EM FACE DO GRANDE NÚMERO DE PROFISSIONAIS ASSOCIADOS QUE ATUAM EM DETERMINADA ESPECIALIDADE. IMPOSSIBILIDADE. (...) 2. Salvo impossibilidade técnica do profissional para exercer os serviços propostos pela coopera- 
tiva, conforme art. $4 .^{\circ}, \mathrm{l}$, da Lei 5.764/71, deve-se considerar ilimitado o número de associados que podem juntar-se ao quadro associativo, face a aplicação do princípio da adesão livre e voluntária que rege o sistema cooperativista. 3 . No caso concreto, a ré aduz que a cooperativa não é obrigada a aceitar todos aqueles que pretendam ingressar na sociedade, podendo deliberar sobre a conveniência e oportunidade da associação de novos médicos, inclusive em face da exceção legal de impossibilidade técnica de prestação de serviços. Contudo, o acórdão recorrido foi claro ao afirmar que a autora possui todas as qualificações necessárias ao exercício de sua especialidade, de modo que não é possível acolher as razões para a negativa de filiação. 4. Recurso especial não conhecido» (REsp n. ${ }^{\circ} 1.124 .273 / \mathrm{CE}$, Rel. Ministro LUIS FELIPE SALOMÃO, Quarta Turma, DJe 19/3/2010).

«DIREITO CIVIL. COOPERATIVA MÉDICA. UNIMED. VEDAÇÃO DE INGRESSO DE NOVOS MÉDICOS. IMPOSSIBILIDADE. 1. Em regra, a limitação ao ingresso de novos associados condiciona-se à impossibilidade técnica do profissional para exercer os serviços propostos pela cooperativa, conforme art. $4^{\circ}$, I, da Lei n..$^{\circ}$.764/71, não bastando a simples alegação de conveniência para os que já integram o quadro de cooperados. 2. No caso concreto, não foi reconhecida pelo acórdão recorrido - sequer acusada pela ré- qualquer inaptidão pessoal dos médicos, ora recorrentes, razão por que se deve franquear-lhes o ingresso na cooperativa médica. 3. Recurso especial provido» (REsp n. ${ }^{\circ}$ 661.292/MG, Rel. Ministro LUIS FELIPE SALOMÃO, Quarta Turma, DJe 8/6/2010).

Em verdade, ao entender que a impossibilidade técnica de prestação de serviços deve ser analisada a luz do candidato à associado, os julgados confundem, ao nosso ver, tal noção ao cumprimento dos requisitos estatutários de ingresso. Dito de outro modo, a (im)possibilidade técnica do profissional trata-se da análise do cumprimento dos requisitos estatutários de ingresso; ultrapassada essa fase, surge para a Cooperativa a faculdade de restringir o ingresso de novos sócios mediante a comprovação de sua incapacidade técnica de prestação de serviços a novos associados.

\section{Conclusões}

As Cooperativas são sociedades que se caracterizam pela propriedade comum e gestão democrática. São abertas a todos os que cumpram os requisitos estatutários de ingresso e que desejem aderir aos propósitos da sociedade. Os valores e princípios que regem tal sociedade tornam inadmissível o estabelecimento de requisitos discriminatórios para a adesão à Cooperativa. 
No Direito brasileiro, a legislação, confere às Cooperativas a prerrogativa de não aceitar novos associados, mesmo diante de candidatos a associados que preencham os requisitos legais e estatutários para associarem-se, sempre que houver «impossibilidade técnica de prestação de serviços».

A análise do discurso decisório do Superior Tribunal de Justiça evidencia o reconhecimento, pela Corte, do Princípio Cooperativista da Adesão Livre e Voluntária, rechaçando o estabelecimento de requisitos discriminatórios e arbitrários como condições de adesão a Cooperativas. Por fim, em relação interpretação acerca da impossibilidade técnica de prestação de serviços pela Cooperativa, a análise de decisões revelou equívocos dos julgadores ao oscilar o entendimento entre a impossibilidade técnica por parte da Cooperativa ou por parte do Profissional.

Entende-se que a interpretação adequada vincula impossibilidade técnica de prestação de serviços à Cooperativa uma vez que relaciona-la ao profissional resta por confundi-la ao cumprimento de requisitos estatutários de ingresso.

\section{Bibliografia}

ALIANÇA COOPERATIVA INTERNACIONAL (ACI). «Guidance Notes to the Cooperative Principles». Acesso em 24 de Maio de 2017. http://ica.coop/en/ blueprint-themes/identity/guidancenotes

ALIANÇA COOPERATIVA INTERNACIONAL (ACI). "Statement on Cooperative Identity». Acesso em 24 de Maio de 2017. http://ica.coop/en/whats-co-op/ co-operative-identity-values-principles

BRASIL. 1971. «LEI 5.764, de 16 de dezembro de 1971». Acesso em 24 de Maio de 2017. http://www.planalto.gov.br/ccivil_03/leis/L5764.htm

ALIANÇA COOPERATIVA INTERNACIONAL (ACI). 1988. "Constituição da República Federativa do Brasil». Acesso em 24 de Maio de 2017. http:// www.planalto.gov.br/ccivil_03/Constituicao/Constituicao.htm

HENRŸ, Hagen. 2012. «Guidelines for cooperative legislation» Geneva: ILO.

ORGANIZAÇÃO DAS NAÇÕES UNIDAS (ONU). «Declaração Universal dos Direitos Humanos» Acesso em 24 de Maio de 2017. http://www.ohchr.org/EN/ Professionallnterest/Pages/InternationalLaw.aspx

ORGANIZAÇÃO INTERNACIONAL DO TRABALHO (OIT). "Recomendação 193/2002». Acesso em 24 de Maio de 2017. http://www.ilo.org/dyn/normlex/en/f?p=NORMLEXPUB:12100:0::NO::P12100_ILO_CODE:R193

SARLET, Ingo Wolfgang. 2012. "Liberdade de associação cooperativa, direitos e garantias fundamentais e relações privadas na perspectiva do Supremo Tribunal Brasileiro». MEIRA, Deolinda Aparício Jurisprudência cooperativa comentada. Lisboa: INCM. 


\section{Derechos de autor}

El Boletín de la Asociación Internacional de Derecho Cooperativo es una revista de acceso abierto lo que significa que es de libre acceso en su integridad inmediatamente después de la publicación de cada número. Se permite su lectura, la búsqueda, descarga, distribución y reutilización legal en cualquier tipo de soporte sólo para fines no comerciales y según lo previsto por la ley; sin la previa autorización de la Editorial (Universidad de Deusto) o el autor, siempre que la obra original sea debidamente citada (número, año, páginas y DOI si procede) y cualquier cambio en el original esté claramente indicado.

\section{Copyright}

The International Association of Cooperative Law Journal is an Open Access journal which means that it is free for full and immediate access, reading, search, download, distribution, and lawful reuse in any medium only for non-commercial purposes, without prior permission from the Publisher or the author; provided the original work is properly cited and any changes to the original are clearly indicated. 